\title{
Higher Accuracy Approximate Solution for Oscillations of a Mass Attached to a Stretched Elastic Wire by Rational Harmonic Balance Method
}

\author{
E. Gimeno, M. L. Álvarez, M. S. Yebra, J. Rosa-Herranz and A. Beléndez \\ Departamento de Física, Ingeniería de Sistemas y Teoría de la Señal. Universidad de \\ Alicante. Apartado 99. E-03080 Alicante. SPAIN
}

E-mail: a.belendez@ua.es, Fax: +34-96-5909750

\begin{abstract}
A second-order modified rational harmonic balance method is used for approximately solve the nonlinear differential equation that governs the oscillations of a system typified as a mass attached to a stretched elastic wire for which the restoring force for this oscillator has an irrational term with a parameter $\lambda$ that characterizes the system. A frequency-amplitude relation is constructed and this frequency is valid for the complete range of oscillation amplitudes $A$ and parameter $\lambda$, and excellent agreement of the approximate frequencies with the exact one is demonstrated and discussed. The discrepancy between the approximate frequency and the exact one never exceed $0.12 \%$. This error corresponds to $\lambda=1$, while for $\lambda<1$ the relative error is much lower. For example, its value is lower than $0.017 \%$ for $\lambda=0.5$.
\end{abstract}

Keywords: Nonlinear oscillator; Analytical approximations; Rational harmonic balance method

\section{Introduction}

Considerable attention has been directed towards the study of nonlinear oscillatory problems in physics and engineering [1,2]. There is a large variety of approximate methods commonly used for solving nonlinear oscillatory systems including perturbations [1-5], variational [6], variational iteration [7-10], homotopy perturbation [11-17] or harmonic balance [2, 18-24] methods, etc. Surveys of the literature with numerous references and useful bibliography and a review of these methods can be found in detail in [2] and [17]. In this paper we apply a modified generalized, rational harmonic balance method to obtain analytic approximate solutions for a nonlinear oscillator typified as a mass attached to a stretched elastic wire. This nonlinear oscillator has been studied by Mickens using the harmonic balance method [2] and it has been recently analyzed by Beléndez et al. using the harmonic balance method [21] and the homotopy perturbation method [25] and by Sun et al. [26] using a
Newton-harmonic balancing approach, by Lan $\mathrm{Xu}$ [27] using He's parameter-expansion method and by Da-Hua Shou [28] using a variational approach. However in most of these papers only the first-order approximation is considered and only Sun et al. [26] take into account higher-order approximations, but they do not solve the nonlinear differential equation directly because they rewrite the second order differential equation in a form that does not contain the square-root expression. We will see that the modified rational harmonic balance method considered in this paper can be applied to nonlinear oscillatory systems where the nonlinear terms are not small and no perturbation parameter is required. In this method the approximate solution obtained approximates all of the harmonics in the exact solution [29], whereas the usual harmonic balance techniques provide an approximation to only the lowest harmonic components. In an attempt to provide better solution methodology a modification in this technique is proposed. The approximate frequency derived here is more 
accurate and closer to the exact solution and the error in the resulting frequency is reduced and the maximum relative error is less than $0.12 \%$ for all values of $A$.

\section{Solution procedure}

Considered a one-dimensional, nonlinear oscillator governed by

$$
\frac{\mathrm{d}^{2} x}{\mathrm{~d} t^{2}}+x-\frac{\lambda x}{\sqrt{1+x^{2}}}=0, \quad 0<\lambda \leq 1
$$

with initial conditions

$$
x(0)=A \quad \text { and } \quad \frac{\mathrm{d} x}{\mathrm{~d} t}(0)=0
$$

Eq. (1) is an example of conservative nonlinear oscillatory system having irrational form for the restoring force and it corresponds to the dimensionless equation of motion of a mass attached to a stretched wire [1]. This is a conservative nonlinear oscillatory system and has the first integral

$$
\frac{1}{2}\left(\frac{\mathrm{d} x}{\mathrm{~d} t}\right)^{2}+V(x)=E \geq 0
$$

where $E$ is the "total energy" of the nonlinear oscillator and the potential function has the irrational form [1]

$$
V(x)=\frac{1}{2} x^{2}-\lambda \sqrt{1+x^{2}}+\lambda
$$

All the motions corresponding to Eq. (1) are periodic [1] and the system will oscillate between the symmetric bounds $[-A, A]$, and the angular frequency and corresponding periodic solution of the nonlinear oscillator are dependent on the amplitude $A$.

For large $x$, and for $0<\lambda \leq 1$,Eq. (1) approximates that of a linear harmonic oscillator

$$
\frac{\mathrm{d}^{2} x}{\mathrm{~d} t^{2}}+x=0 \text { for } x>>1 \text { and } 0<\lambda \leq 1
$$

so, for large $A$, we have $\omega \approx 1$. For small $x$, and for $0<\lambda<1$, the equation of motion also approximates that of a linear oscillator

$$
\frac{\mathrm{d}^{2} x}{\mathrm{~d} t^{2}}+(1-\lambda) x=0 \text { for } x<<1 \text { and } 0<\lambda<1
$$

and $\omega \approx \sqrt{1-\lambda}$ for small $A$. However, for small $x$, and for $\lambda=1$, Eq. (1) approximates that of a truly nonlinear cubic oscillator

$$
\frac{\mathrm{d}^{2} x}{\mathrm{~d} t^{2}}+\frac{1}{2} x^{3}=0 \text { for } x<<1 \text { and } \lambda=1
$$

and $\omega \approx 0.59907 A$ [2], which tends to zero when $A$ decreases. Consequently the angular frequency $\omega$ increases from $\sqrt{1-\lambda}$ to 1 as the initial value of $x(0)=A$ increases.

Eq. (1) is not amenable to exact treatment and, therefore, approximate techniques must be resorted to.

The main objective of this paper is to solve Eq. (1) by applying a modified rational harmonic balance method (RHBM) introduced by Beléndez et al. [30]. A new independent variable $\tau=\omega t$ is introduced. Then Eqs. (1) and (2) can be rewritten as

$$
\begin{gathered}
\omega^{2} \frac{\mathrm{d}^{2} x(\tau)}{\mathrm{d} \tau^{2}}+x(\tau)-\frac{\lambda x(\tau)}{\sqrt{1+x^{2}(\tau)}}=0 \\
x(0)=A, \frac{\mathrm{d} x}{\mathrm{~d} \tau}(0)=0
\end{gathered}
$$

The new independent variable is chosen in such a way that the solution of Eq. (8) is a periodic function of $\tau$ of period $2 \pi$. The angular frequency of the nonlinear oscillator is $\omega$ and it is a function of the initial amplitude $A$. The corresponding period of the nonlinear oscillation is given by $T=2 \pi / \omega$. Both the periodic solution $x(t)$ and frequency $\omega$ (thus period $T$ ) depends on $A$. In order to determine an approximate solution, we use a generalized, rational form given by the following expression $[1,29]$

$$
x_{2}(\tau)=\frac{A_{1} \cos \tau}{1+B_{2} \cos 2 \tau}
$$

In this equation $A_{1}, B_{2}$ and $\omega$ are to be determined as functions of the initial conditions expressed in Eq. (9) and $\left|B_{2}\right|<<1$. From Eq. (9) 
we obtain $A_{1}=\left(1+B_{2}\right) A$ and Eq. (10) can be rewritten as follows

$$
x_{2}(\tau)=\frac{A\left(1+B_{2}\right) \cos \tau}{1+B_{2} \cos 2 \tau}
$$

Substituting Eq. (11) into Eq. (8) gives

$$
\begin{aligned}
& \left(-\omega^{2}+1\right) \frac{A\left(1+B_{2}\right) \cos \tau}{1+B_{2} \cos 2 \tau}+\omega^{2} \frac{4 A B_{2}\left(1+B_{2}\right) \cos 3 \tau}{\left(1+B_{2} \cos 2 \tau\right)^{2}} \\
& +\omega^{2} \frac{8 A B_{2}^{2}\left(1+B_{2}\right) \cos \tau \sin ^{2} 2 \tau}{\left(1+B_{2} \cos 2 \tau\right)^{3}} \\
& -\frac{\lambda A\left(1+B_{2}\right) \cos \tau}{\sqrt{\left(1+B_{2} \cos 2 \tau\right)^{2}+A^{2}\left(1+B_{2}\right)^{2} \cos ^{2} \tau}}=0
\end{aligned}
$$

Eq. (12) can be written as follows

$$
F\left(A, B_{2}, \omega, \tau\right)=0
$$

As $\left|B_{2}\right|<<1$ we can do the following Taylor series expansion

$$
F\left(A, B_{2}, \omega, \tau\right)=\sum_{n=0}^{\infty} f_{n}(A, \omega, \tau) B_{2}^{n}
$$

where

$$
f_{n}(A, \omega, \tau)=\frac{1}{n !}\left(\frac{\partial^{n} F\left(A, B_{2}, \omega, \tau\right)}{\partial B_{2}^{n}}\right)_{B_{2}=0}
$$

Before applying the harmonic balancing to Eq. (12), and considering that $\left|B_{2}\right|<<1$, we consider the following approximation in Eqs. (13) and (14)

$$
\begin{aligned}
& F\left(A, B_{2}, \omega, \tau\right) \\
& \quad \approx f_{0}(A, \omega, \tau)+f_{1}(A, \omega, \tau) B_{2}+f_{2}(A, \omega, \tau) B_{2}^{2}=0
\end{aligned}
$$

Expanding Eq. (16) in a trigonometric series yields

$$
H_{1}\left(A, B_{2}, \omega\right) \cos \tau+H_{3}\left(A, B_{2}, \omega\right) \cos 3 \tau+H O H=0
$$

where

$$
H_{1}\left(A, B_{2}, \omega\right)=\frac{4}{\pi} \int_{0}^{\pi / 2}\left(f_{0}+f_{1} B_{2}+f_{2} B_{2}^{2}\right) \cos \tau d \tau
$$

$$
H_{3}\left(A, B_{2}, \omega\right)=\frac{4}{\pi} \int_{0}^{\pi / 2}\left(f_{0}+f_{1} B_{2}+f_{2} B_{2}^{2}\right) \cos 3 \tau d \tau
$$

Setting the coefficients of $\cos \tau$ and $\cos 3 \tau$ to zero in Eq. (17) we can obtain $B_{2}$ and the second order approximate frequency $\omega$ as a function of A. From Eqs. (12)-(19) we obtain

$$
\begin{aligned}
& H_{1}\left(A, B_{2}, \omega\right)=\frac{1}{6 \pi A^{5}}\left\{-3 \pi A^{6}\left(2+B_{2}\right)\left(-1+\omega^{2}\right)\right. \\
& \quad-8 \lambda\left[3 A^{4}+64 B_{2}^{2}+4 A^{2} B_{2}\left(8 B_{2}-3\right)\right] E\left(-A^{2}\right) \\
& +8 \lambda\left[3 A^{4}-6 A^{2}\left(2+A^{2}\right) B_{2}\right. \\
& \left.\left.\quad+4\left(4+A^{2}\right)\left(4+3 A^{2}\right) B_{2}^{2}\right] K\left(-A^{2}\right)\right\}=0
\end{aligned}
$$

$$
\begin{aligned}
& H_{3}\left(A, B_{2}, \omega\right)=\frac{1}{60 \pi A^{7}}\left\{15 \pi A^{8} B_{2}\left(2+B_{2}\right)\left(-1+9 \omega^{2}\right)\right. \\
& +16 \lambda\left[5 A^{6}+2304 B_{2}^{2}+64 A^{2} B_{2}\left(41 B_{2}-5\right)\right. \\
& \left.+4 A^{4}\left(136 B_{2}^{2}-55 B_{2}+10\right)\right] E\left(-A^{2}\right) \\
& \quad-16 \lambda\left[2304 B_{2}^{2}+64 A^{2} B_{2}\left(59 B_{2}-5\right)\right. \\
& +5 A^{6}\left(36 B_{2}^{2}-18 B_{2}+5\right) \\
& \left.\left.+4 A^{4}\left(428 B_{2}^{2}-95 B_{2}+10\right)\right] K\left(-A^{2}\right)\right\}=0
\end{aligned}
$$

where $K(m)$ and $E(m)$ are the complete elliptic integrals of the first and second kind, respectively, defined as follows

$$
\begin{aligned}
& K(m)=\int_{0}^{\pi / 2} \frac{d \theta}{\sqrt{1-m \sin ^{2} \theta}} \\
& E(m)=\int_{0}^{\pi / 2} \sqrt{1-m \sin ^{2} \theta} d \theta
\end{aligned}
$$

Solving Eq. (20) for $\omega$ yields 


$$
\begin{aligned}
& \omega_{2}(A)= \\
& =\left[1+\frac{8 \lambda}{3 \pi A^{6}\left(2+B_{2}\right)}\left\{\left[3 A^{4}-\left(12 A^{2}+6 A^{4}\right) B_{2}\right.\right.\right. \\
& \left.+\left(64+64 A^{2}+12 A^{4}\right) B_{2}^{2}\right] K\left(-A^{2}\right) \\
& \left.\left.-\left[3 A^{4}-12 A^{2} B_{2}+\left(64+32 A^{2}\right) B_{2}^{2}\right] E\left(-A^{2}\right)\right\}\right]^{1 / 2}
\end{aligned}
$$

and substituting Eq. (24) into Eq. (21) gives the following cubic equation which must be solved to obtain $B_{2}$

$$
B_{2}^{3}+a_{2}(A) B_{2}^{2}+a_{1}(A) B_{2}+a_{0}(A)=0
$$

where

$$
a_{j}(A)=\frac{b_{j}(A)}{b_{3}(A)}, \quad j=0,1,2
$$

and

$$
\begin{aligned}
& b_{0}(A)= \\
& \quad=10 \lambda A^{4}\left[\left(8+A^{2}\right) E\left(-A^{2}\right)-\left(8+5 A^{2}\right) K\left(-A^{2}\right)\right]
\end{aligned}
$$

$$
\begin{aligned}
& b_{1}(A)=30 \pi A^{8} \\
& \quad-5 \lambda A^{2}\left[\left(128+88 A^{2}+27 A^{4}\right) E\left(-A^{2}\right)\right. \\
& \left.-\left(128+152 A^{2}+63 A^{4}\right) K\left(-A^{2}\right)\right]
\end{aligned}
$$

$b_{2}(A)=15 \pi A^{8}$

$+2 \lambda\left[\left(2304+2624 A^{2}+814 A^{4}\right) E\left(-A^{2}\right)\right.$$$
\left.-\left(2304+3776 A^{2}+1982 A^{4}+315 A^{6}\right) K\left(-A^{2}\right)\right]
$$

$$
\begin{gathered}
b_{3}(A)=180 \lambda A^{2}\left[\left(-16-8 A^{2}\right) E\left(-A^{2}\right)\right. \\
\left.+\left(16+16 A^{2}+3 A^{4}\right) K\left(-A^{2}\right)\right]
\end{gathered}
$$

The polynomial discriminant, $D(A)$, of Eq. (25) is defined as follows [1]

$$
D(A)=\left[\frac{p(A)}{3}\right]^{3}+\left[\frac{q(A)}{2}\right]^{2}
$$

where

$$
p=\frac{3 a_{1}-a_{2}^{2}}{3}
$$

$$
q=\frac{9 a_{1} a_{2}-27 a_{0}-2 a_{2}^{3}}{27}
$$

Determining which roots of Eq. (25) are real and which are complex can be accomplished by noting that if the polynomial discriminant $D>0$, one root is real and two are complex conjugates; if $D=0$, all roots are real and at least two are equal; and if $D<0$, all roots are real and unequal [1]. As we can see in Eq. (24), for $B_{2}=0$, the approximate frequency for the first-order harmonic balance (HBM) approximation is obtained

$$
\omega_{1}(A)=\sqrt{1+\frac{4 \lambda}{\pi A^{2}}\left[K\left(-A^{2}\right)-E\left(-A^{2}\right)\right]}
$$

Therefore, the second approximation to the periodic solution of the nonlinear oscillator is given by the following equation

$$
x_{2}(t)=\frac{A\left(1+B_{2}\right) \cos \omega_{2} t}{1+B_{2} \cos 2 \omega_{2} t}
$$

This periodic solution has the following Fourier series expansion

$$
x_{2}(t)=\sum_{n=0}^{\infty} a_{2 n+1} \cos \left[(2 n+1) \omega_{2} t\right]
$$

where [30]

$$
\begin{aligned}
& a_{2 n+1}= \\
& =(-1)^{n} 2^{n+1} A \sqrt{\frac{1+B_{2}}{1-B_{2}}}\left(\frac{B_{2}}{1-B_{2}}\right)^{n}\left(\frac{\sqrt{1-B_{2}}}{\sqrt{1-B_{2}}+\sqrt{1+B_{2}}}\right)^{2 n+1}
\end{aligned}
$$

As we can see, Eq. (35) gives an expression that approximates all of the harmonics in the exact solution whereas the usual harmonic balancing techniques provide and approximation to only the lowest harmonic components.

\section{Results and discussion}

\subsection{Comparison with the exact and other approximate solutions}

We illustrate the accuracy of the proposed approach by comparing the approximate frequency $\omega_{2}(A)$ obtained in this paper with 
the exact frequency $\omega_{e}(A)$ and other results in the literature. The calculation of the exact angular frequency, $\omega_{e}(A)$, proceeds as follows. A first integral of Eq. (1) is [1]

$$
\frac{1}{2}\left(\frac{\mathrm{d} x}{\mathrm{~d} t}\right)^{2}+\frac{1}{2} x^{2}-\lambda \sqrt{1+x^{2}}=\frac{1}{2} A^{2}-\lambda \sqrt{1+A^{2}}
$$

where we are taken into account the initial conditions in Eq. (2). We can derive the exact frequency as follows

$$
\begin{aligned}
& \omega_{e}(A)= \\
& =\frac{\pi}{2 A}\left[\int_{0}^{1} \frac{d u}{\sqrt{A^{2}\left(1-u^{2}\right)-2 \lambda\left(\sqrt{1+A^{2}}-\sqrt{1+A^{2} u^{2}}\right)}}\right]^{-1}
\end{aligned}
$$

Sun et al [26] rewrote Eq. (1) as follows

$$
\left(1+x^{2}\right)\left(\frac{\mathrm{d}^{2} x}{\mathrm{~d} t^{2}}+x\right)^{2}-\lambda^{2} x^{2}=0
$$

and applying a linearized harmonic balance method they obtained the following second-order approximate frequency

$$
\begin{aligned}
& \omega_{2 S W L}(A)= \\
& =\sqrt{1-\frac{2 \lambda}{\sqrt{4+3 A^{2}}}+\frac{7 \lambda^{2}-4 \lambda \sqrt{4+3 A^{2}}}{2 F(A) \sqrt{4+3 A^{2}}} A^{4}}
\end{aligned}
$$

where

$$
\begin{aligned}
F(A)= & 2\left(16+20 A^{2}+5 A^{4}\right) \sqrt{4+3 A^{2}} \\
& -\lambda\left(64+78 A^{2}+19 A^{4}\right)
\end{aligned}
$$

Comparison of the exact frequency $\omega_{e}(A)$ obtained by integrating Eq. (39), with the proposed frequency $\omega_{2}(A)$ computed using Eqs. (24) and (25) and the second-order approximate frequency $\omega_{2 S W L}(A)$ (Eqs. (41) and (42)) is shown in Tables $1-5$ for $=0.1,0.5$, $0.75,0.95$ and 1 , respectively. In these tables the values of $B_{2}$ and the sign of the discriminant $D$ (Eq. (31)) have been also included. The percentage errors $(P E)$ have been computed by using the equation

$$
P E \text { of } \omega_{j}(\%)=100\left|\frac{\omega_{j}-\omega_{e}}{\omega_{e}}\right|, \quad j=1,2
$$

From Tables 1-5, it can be observed that Eq. (24) yields excellent analytical approximate frequencies both for small and large values of oscillation amplitude $A$ and parameter $\lambda$.

Furthermore, it has shown that the analytical approximate frequency in Eq. (24) is more accurate that that in Eq. (34). We have

$\lim _{A \rightarrow 0} B_{2}(A)=0, \quad \lim _{A \rightarrow 0} \frac{\omega_{1}(A)}{\omega_{e}(A)}=\lim _{A \rightarrow 0} \frac{\omega_{2}(A)}{\omega_{e}(A)}=1$

for $0<\lambda<1$, and

$\lim _{A \rightarrow \infty} B_{2}(A)=0, \quad \lim _{A \rightarrow \infty} \frac{\omega_{1}(A)}{\omega_{e}(A)}=\lim _{A \rightarrow \infty} \frac{\omega_{2}(A)}{\omega_{e}(A)}=1$

for $0<\lambda \leq 1$.

For $\lambda=1$ and small values of $A$ it is possible to do the power series expansion of Eq. (25) and we obtain

$$
\begin{aligned}
\left(\frac{4}{27}+\frac{16}{9}\right. & \left.B_{2}+\frac{14}{9} B_{2}^{2}+B_{2}^{3}\right)+ \\
& +\left(\frac{5}{108}+\frac{25}{24} B_{2}+\frac{35}{48} B_{2}^{2}\right) A^{2}+\ldots \approx 0
\end{aligned}
$$

Solving Eq. (46) for and expanding the result in power series for small $A$, yields

$$
B_{2} \approx-0.0900126+0.0273046 A^{2}+\ldots
$$

Substituting Eq. (47) into Eq. (24) and doing the power series expansion for small values of $A$, the following equation can be obtained

$$
\omega_{2}(A) \approx 0.599098 A+\ldots \quad(\lambda=1)
$$

For small values of the oscillation amplitude, $A$, we can also obtain

$$
\omega_{e}(A) \approx \frac{\pi A}{4 K(-1)} \approx 0.599070 A+\ldots \quad(\lambda=1)
$$


Table 1 Comparison of the approximate frequencies with exact frequency when $\lambda=0.1$ and values of the parameter $B_{2}$

\begin{tabular}{lcclcrl}
\hline$A$ & $\omega_{e}$ & $\begin{array}{c}\omega_{2} \\
\text { Eq. }(24)\end{array}$ & $\begin{array}{l}\text { Relative } \\
\text { error }(\%)\end{array}$ & $B_{2}(D)$ & $\begin{array}{c}\omega_{2 S W L} \\
\text { Eq. }(41)\end{array}$ & $\begin{array}{l}\text { Relative } \\
\text { error }(\%)\end{array}$ \\
\hline 0.1 & 0.94887970 & 0.94887969 & 0.000000085 & $-0.000034366(<0)$ & 0.94887965 & 0.0000050 \\
0.4 & 0.95155713 & 0.95155712 & 0.00000061 & $-0.000479679(<0)$ & 0.95154901 & 0.00085 \\
0.7 & 0.95613672 & 0.95613656 & 0.000017 & $-0.00113751(<0)$ & 0.95608678 & 0.0052 \\
1 & 0.96109793 & 0.96109734 & 0.000061 & $-0.00169681(<0)$ & 0.96097722 & 0.013 \\
4 & 0.98507517 & 0.98507342 & 0.00018 & $-0.00194353(<0)$ & 0.98491783 & 0.016 \\
7 & 0.99112293 & 0.99112209 & 0.000085 & $-0.00132827(<0)$ & 0.99106984 & 0.0054 \\
10 & 0.99371282 & 0.99371236 & 0.000047 & $-0.000985555(<0)$ & 0.99369292 & 0.0020 \\
\hline
\end{tabular}

Table 2 Comparison of the approximate frequencies with exact frequency when $\lambda=0.5$ and values of the parameter $B_{2}$

\begin{tabular}{lcrlcrl}
\hline \multirow{2}{*}{$A$} & $\omega_{e}$ & $\begin{array}{c}\omega_{2} \\
\text { Eq. }(24)\end{array}$ & $\begin{array}{l}\text { Relative } \\
\text { error }(\%)\end{array}$ & $B_{2}(D)$ & $\begin{array}{c}\omega_{2 S W L} \\
\text { Eq. }(41)\end{array}$ & $\begin{array}{l}\text { Relative } \\
\text { error }(\%)\end{array}$ \\
\hline 0.1 & 0.70842299 & 0.70842295 & 0.0000055 & $-0.000308494(<0)$ & 0.70842275 & 0.000034 \\
0.4 & 0.72612552 & 0.72612495 & 0.000078 & $-0.00412723(<0)$ & 0.72608394 & 0.0057 \\
0.7 & 0.75547954 & 0.75547104 & 0.0011 & $-0.00915932(<0)$ & 0.75522825 & 0.033 \\
1 & 0.78617121 & 0.78614353 & 0.0035 & $-0.0127928(<0)$ & 0.78557662 & 0.076 \\
4 & 0.92272685 & 0.92267145 & 0.0060 & $-0.0112367(<0)$ & 0.92206265 & 0.072 \\
7 & 0.95468855 & 0.95466418 & 0.0026 & $-0.00723305(<0)$ & 0.95449238 & 0.021 \\
10 & 0.96810224 & 0.96808944 & 0.0013 & $-0.00523299(<0)$ & 0.96804316 & 0.0061 \\
\hline
\end{tabular}

Table 3 Comparison of the approximate frequencies with exact frequency when $\lambda=0.75$ and values of the parameter $B_{2}$

\begin{tabular}{lcclcrrl}
\hline \multirow{2}{*}{$A$} & $\omega_{e}$ & $\begin{array}{c}\omega_{2} \\
\text { Eq. }(24)\end{array}$ & $\begin{array}{l}\text { Relative } \\
\text { error }(\%)\end{array}$ & \multicolumn{2}{c}{$B_{2}(D)$} & $\begin{array}{c}\omega_{2 S W L} \\
\text { Eq. }(41)\end{array}$ & $\begin{array}{l}\text { Relative } \\
\text { error }(\%)\end{array}$ \\
\hline 0.1 & 0.50278606 & 0.50278601 & 0.0000098 & $-0.000918948(<0)$ & 0.50278586 & 0.000039 \\
0.4 & 0.53921331 & 0.53921002 & 0.00061 & -0.0112708 & $(<0)$ & 0.53917637 & 0.0069 \\
0.7 & 0.59627838 & 0.59623848 & 0.0067 & $-0.0222423(>0)$ & 0.59600871 & 0.0045 \\
1 & 0.65277100 & 0.65265815 & 0.017 & -0.0281667 & $(>0)$ & 0.65207056 & 0.11 \\
4 & 0.88125457 & 0.88110668 & 0.017 & $-0.0186939(<0)$ & 0.88043574 & 0.093 \\
7 & 0.93109439 & 0.93103405 & 0.0065 & $-0.0114925(<0)$ & 0.93088145 & 0.023 \\
10 & 0.95169604 & 0.95166527 & 0.0032 & $-0.00816673(<0)$ & 0.95165150 & 0.0047 \\
\hline
\end{tabular}

Table 4 Comparison of the approximate frequencies with exact frequency when $\lambda=0.95$ and values of the parameter $B_{2}$

\begin{tabular}{lcrlrrrl}
\hline \multirow{2}{*}{$A$} & $\omega_{e}$ & $\begin{array}{c}\omega_{2} \\
\text { Eq. }(24)\end{array}$ & $\begin{array}{l}\text { Relative } \\
\text { error }(\%)\end{array}$ & \multicolumn{2}{c}{$B_{2}(D)$} & $\begin{array}{c}\omega_{2 S W L} \\
\text { Eq. }(41)\end{array}$ & $\begin{array}{l}\text { Relative } \\
\text { error }(\%)\end{array}$ \\
\hline 0.1 & 0.23136709 & 0.23136702 & 0.000027 & -0.00550941 & $(<0)$ & 0.23137256 & 0.0024 \\
0.4 & 0.31764173 & 0.31761160 & 0.0095 & -0.0417710 & $(>0)$ & 0.31801743 & 0.12 \\
0.7 & 0.42643080 & 0.42624370 & 0.044 & -0.0562026 & $(>0)$ & 0.42697924 & 0.13 \\
1 & 0.52033467 & 0.51995040 & 0.074 & -0.0573559 & $(>0)$ & 0.52041800 & 0.016 \\
4 & 0.84639887 & 0.88461221 & 0.033 & -0.0259595 & $(<0)$ & 0.84561002 & 0.093 \\
7 & 0.91171181 & 0.91160677 & 0.012 & -0.0152847 & $(<0)$ & 0.91153912 & 0.019 \\
10 & 0.93833273 & 0.93828057 & 0.0056 & -0.0106911 & $(<0)$ & 0.93832610 & 0.00071 \\
\hline
\end{tabular}


Table 5 Comparison of the approximate frequencies with exact frequency when $\lambda=1$ and values of the parameter $B_{2}$

\begin{tabular}{lcclcrrl}
\hline \multirow{2}{*}{$A$} & $\omega_{e}$ & $\begin{array}{c}\omega_{2} \\
\text { Eq. }(24)\end{array}$ & $\begin{array}{l}\text { Relative } \\
\text { error }(\%)\end{array}$ & \multicolumn{2}{c}{$B_{2}(D)$} & $\begin{array}{c}\omega_{2 S W L} \\
\text { Eq. }(41)\end{array}$ & $\begin{array}{l}\text { Relative } \\
\text { error }(\%)\end{array}$ \\
\hline 0.1 & 0.05973045 & 0.05973115 & 0.0012 & -0.0897435 & $(>0)$ & 0.06026085 & 0.89 \\
0.4 & 0.22916187 & 0.22907153 & 0.040 & -0.0859630 & $(>0)$ & 0.23078447 & 0.71 \\
0.7 & 0.37081339 & 0.37048459 & 0.088 & -0.0791806 & $(<0)$ & 0.37238041 & 0.42 \\
1 & 0.48085077 & 0.48029191 & 0.12 & -0.0713778 & $(>0)$ & 0.48163170 & 0.16 \\
4 & 0.83742053 & 0.83710095 & 0.038 & -0.0280031 & $(>0)$ & 0.83666882 & 0.090 \\
7 & 0.90679079 & 0.90667190 & 0.013 & -0.0162932 & $(<0)$ & 0.90663702 & 0.017 \\
10 & 0.93495724 & 0.93489862 & 0.0063 & -0.0113491 & $(<0)$ & 0.93496420 & 0.00074 \\
\hline
\end{tabular}

From Eqs. (24), (47), (48) and (49) it follows that (for $\lambda=1$ )

$$
\begin{gathered}
\lim _{A \rightarrow 0} B_{2}(A)=-0.0900126 \\
\lim _{A \rightarrow 0} \frac{\omega_{1}(A)}{\omega_{e}(A)}=1.0222 \\
\lim _{A \rightarrow 0} \frac{\omega_{2}(A)}{\omega_{e}(A)}=\frac{0.599098}{0.599070}=1.000047
\end{gathered}
$$

From Eq. (52) we can see that the relative error is $0.0047 \%$ when $A$ approaches zero.
For $A=1$ and $\lambda=0.1,0.5,0.75,0.95$ and 1 , the (numerical) exact periodic solution obtained by numerically integrating Eq. (1), the approximate periodic solution $x_{1}(t)$ computed by $A \cos \omega_{1} t$ (where $\omega_{1}$ is given by Eq. (34)) and the approximate periodic solution $x_{2}(t)$ computed by Eq. (35), are plotted in Figures 1-5 as a function of $h=\omega_{e} t /(2 \pi)$.

These figures show that the proposed solutions in Eqs. (34) and (35) are in excellent agreement with exact periodic solutions for the whole range of values of $\lambda$.

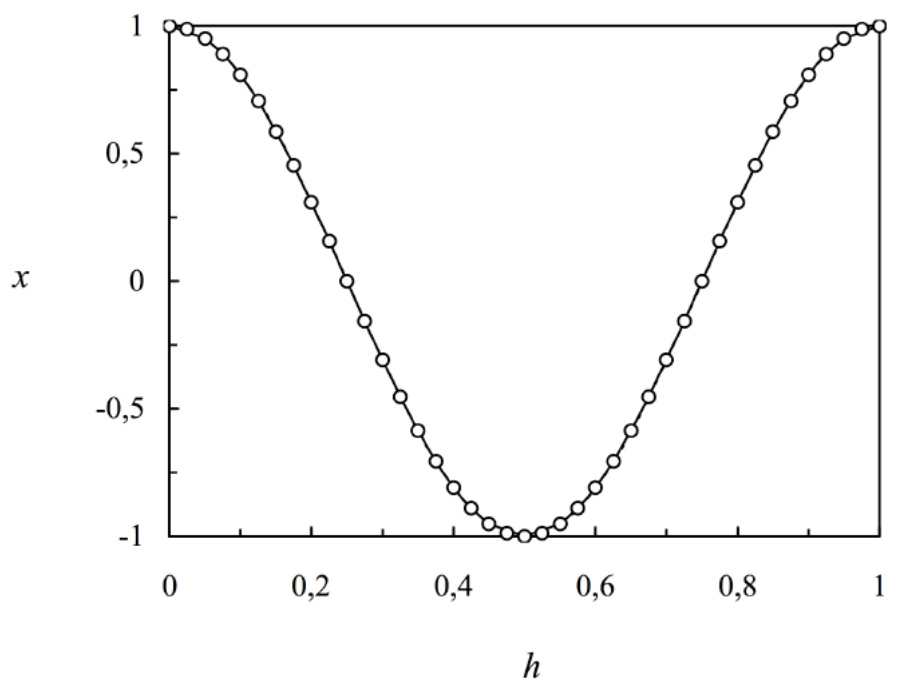

Fig. 1 Comparison of first order solution obtained by the HBM (dashed line), second order solution obtained using the RHBM (circles) with the exact solution (continuous line) for $\lambda=0.1$ and $A=1$. 


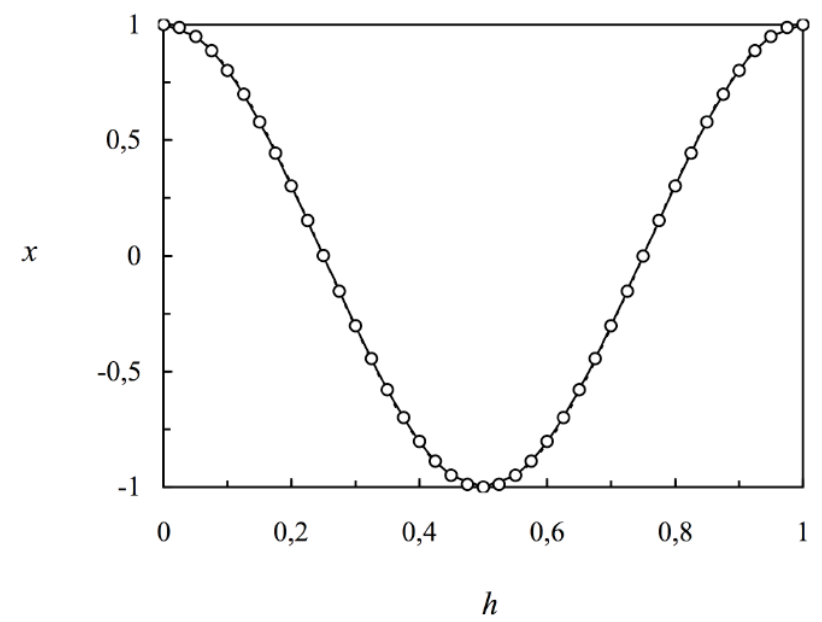

Fig. 2 Comparison of first order solution obtained by the HBM (dashed line), second order solution obtained using the RHBM (circles) with the exact solution (continuous line) for $\lambda=0.5$ and $A=1$.

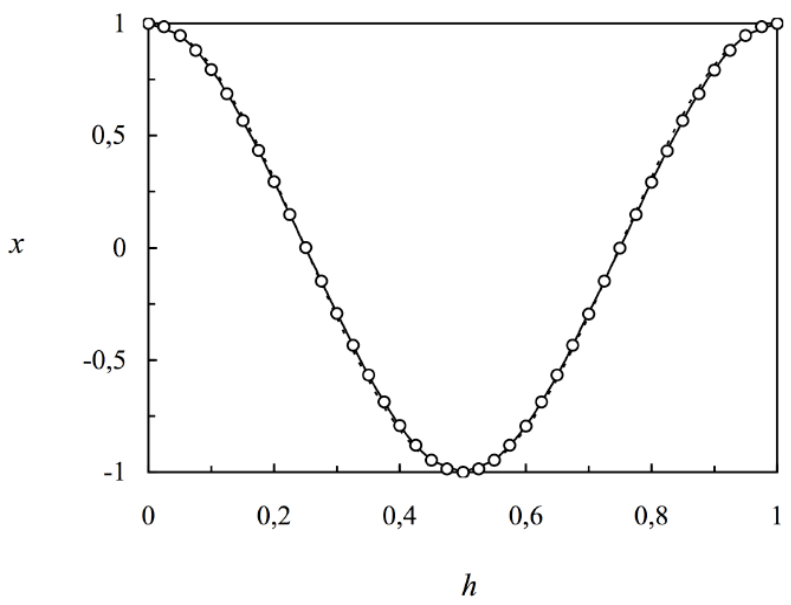

Fig. 3 Comparison of first order solution obtained by the HBM (dashed line), second order solution obtained using the RHBM (circles) with the exact solution (continuous line) for $\lambda=0.75$ and $A=1$.

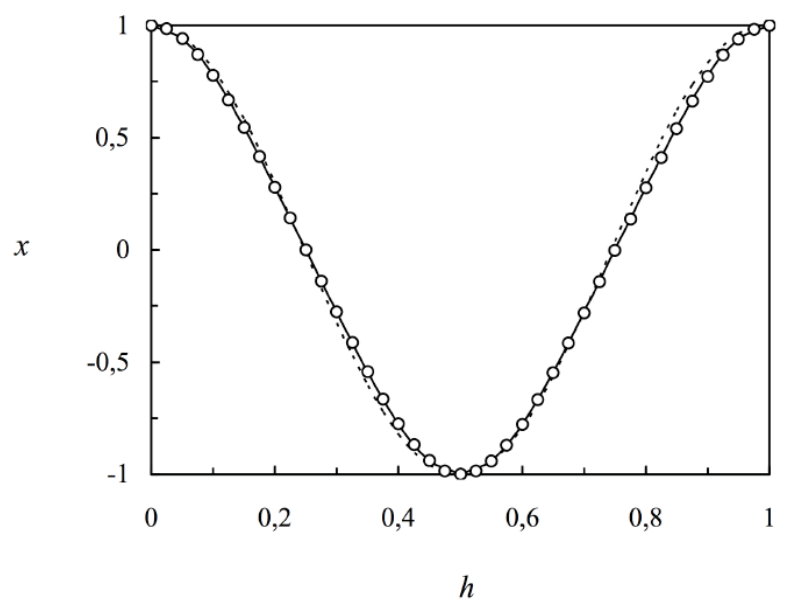

Fig. 4 Comparison of first order solution obtained by the HBM (dashed line), second order solution obtained using the RHBM (circles) with the exact solution (continuous line) for $\lambda=0.95$ and $A=1$. 


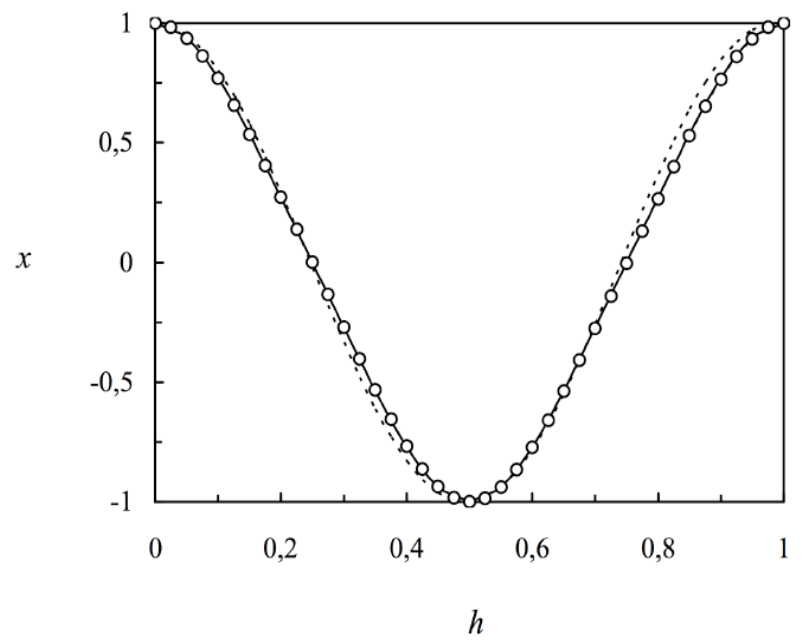

Fig. 5 Comparison of first order solution obtained by the HBM (dashed line), second order solution obtained using the RHBM (circles) with the exact solution (continuous line) for $\lambda=1$ and $A=1$.

\subsection{An alternative approximate solution for $B_{2}$}

Now we are going to considerer a simpler expression for $B_{2}$ and then for $\omega_{2}$. Eq. (25) is cubic and therefore has three solutions. However, the root of interest is one for which $\left|B_{2}\right|<<1$. To proceed, assume that such a solution, $B_{2}$, exists (this is not actually an assumption, because we have seen in section 2 that $B_{2}$ exists and its value is lower than one).

A first approximate value $B_{2}^{(1)}$ can be determined by neglecting the first two terms in Eq. (25)

$$
a_{1}(A) B_{2}^{(1)}+a_{0}(A)=0
$$

Solving this equation gives

$$
B_{2}^{(1)}=-a_{0} / a_{1}
$$

To further improve this result, we assume that $B_{2}$ can be written as follows

$$
B_{2}=B_{2}^{(1)}+\delta
$$

where $\delta$ is a correction term and $|\delta|<<\left|B_{2}^{(1)}\right|$. Substituting Eq. (55) into Eq. (25) and linearizing with respect to the correction term $\delta$ lead to

$$
\begin{array}{r}
{\left[B_{2}^{(1)}\right]^{3}\left(1+\frac{3 \delta}{B_{2}^{(1)}}\right)+a_{2}\left[B_{2}^{(1)}\right]^{2}\left(1+\frac{2 \delta}{B_{2}^{(1)}}\right)+} \\
+a_{1} B_{2}^{(1)}\left(1+\frac{\delta}{B_{2}^{(1)}}\right)+a_{0}=0
\end{array}
$$

which is a linear equation instead $d$ of a cubic equation. Solving Eq. (56) it follows that

$$
\begin{aligned}
B_{2}=-\frac{a_{0}\left(2 a_{0}^{2}+a_{1}^{3}-a_{0} a_{1} a_{2}\right)}{a_{1}\left(3 a_{0}^{2}+a_{1}^{3}-2 a_{0} a_{1} a_{2}\right)} \\
=-\frac{b_{0}\left(2 b_{0}^{2} b_{3}+b_{1}^{3}-b_{0} b_{1} b_{2}\right)}{b_{1}\left(3 b_{0}^{2} b_{3}+b_{1}^{3}-2 b_{0} b_{1} b_{2}\right)}
\end{aligned}
$$

where Eq. (54) has been taken into account and $b_{0}, b_{1}, b_{2}$ and $b_{3}$ are given in Eqs. (27)-(30). Substituting Eq. (57) into Eq. (24) we obtain the value $\omega_{2}$ of as a function of $\lambda$ and $A$.

For small values of $A$ it is possible to do the power-series expansion of the approximate new angular frequency $\omega_{2}$ and the following equations can be obtained

$$
\begin{aligned}
\omega_{2}(A) \approx & \sqrt{1-\lambda}+\frac{3 \lambda}{16 \sqrt{1-\lambda}} A^{2}+\frac{3 \lambda(33 \lambda-40)}{1024(1-\lambda)^{3 / 2}} A^{4} \\
& +\frac{\lambda\left(1031 \lambda^{2}-2350 \lambda+1400\right)}{16384(1-\lambda)^{5 / 2}} A^{6}+\ldots
\end{aligned}
$$

for $0<\lambda<1$, and 


$$
\begin{aligned}
\omega_{2}(A) & \approx \frac{1}{4} \sqrt{\frac{29104261}{5067965}} A \\
& -\frac{2263791463341459 A^{3}}{1037919232 \sqrt{147499376098865}}+\ldots \\
& =0.599103 A-0.179588 A^{3}+\ldots
\end{aligned}
$$

for $\lambda=1$. For small values of the amplitude $A$ it is possible to take into account the following approximation for the exact frequency (Eq. (39)) which is valid for $0<\lambda<1$

$$
\begin{aligned}
\omega_{e}(A) & \approx \frac{\pi}{2}\left[\int _ { 0 } ^ { 1 } \frac { d u } { \sqrt { 1 - u ^ { 2 } } } \left(\frac{1}{\sqrt{1-\lambda}}-\frac{\lambda\left(1+u^{2}\right)}{8(1-\lambda)^{3 / 2}} A^{2}\right.\right. \\
& \left.\left.-\frac{\lambda\left[2(\lambda-4) u^{2}+(5 \lambda-8)\left(1+u^{4}\right)\right]}{128(1-\lambda)^{5 / 2}} A^{4}+\ldots\right)\right]^{-1}
\end{aligned}
$$

and the following frequency for $\lambda=1$

$$
\begin{aligned}
& \omega_{e}(A) \\
& \approx \frac{\pi}{2}\left[\int_{0}^{1}\left(\frac{2}{A \sqrt{1-u^{4}}}+\frac{\left(1+u+u^{2}\right) A}{2\left(1+u^{2}\right) \sqrt{1-u^{4}}}+\ldots\right) d u\right]^{-1}
\end{aligned}
$$

From Eqs. (60) and (61), the power-series expansions of the exact angular frequencies for small $A$ are

$$
\begin{aligned}
& \omega_{e}(A) \approx \sqrt{1-\lambda}+\frac{3 \lambda}{16 \sqrt{1-\lambda}} A^{2}+ \\
& +\frac{3 \lambda(33 \lambda-40)}{1024(1-\lambda)^{3 / 2}} A^{4} \\
& +\frac{\lambda\left(1025 \lambda^{2}-2344 \lambda+1400\right)}{16384(1-\lambda)^{5 / 2}} A^{6}+\ldots
\end{aligned}
$$

for $0<\lambda<1$, and for $\lambda=1$ we obtain

$$
\begin{aligned}
\omega_{e}(A) & \approx \frac{\pi}{4 K(-1)} A \\
+ & \frac{\pi}{2}\left[\frac{1}{8 K(-1)}-\frac{3 E(-1)}{16 K^{2}(-1)}\right] A^{3}+\ldots \\
& =0.599070 A-0.177537 A^{3}+\ldots
\end{aligned}
$$

As can be seen, in the expansion of the angular frequency for $0<\lambda<1$ (Eq. (58)), the first three terms are the same as the first three terms of the equation obtained in the power-series expansion of the exact angular frequency (Eq. (62)). Whereas, if we compare the fourth term in Eq. (58) with the fourth term in the series expansion of the exact frequency (Eq. (62)), we can see that the relative error of the fourth term of the series expansion of $\omega_{2}(A)$ is

$$
\varepsilon(\lambda)=\frac{6 \lambda(1-\lambda)}{1400-2344 \lambda+1025 \lambda^{2}}
$$

which is lower than $0.54 \%$ for $0<\lambda<1$.

Comparing Eqs. (59) and (63) for $\lambda=1$ we obtain

$$
\lim _{A \rightarrow 0} \frac{\omega_{2}}{\omega_{e}}=\frac{1}{4} \sqrt{\frac{29104261}{5067965}} \frac{4 K(-1)}{\pi}=1.000055(65)
$$

We can see that the relative error is $0.0055 \%$ when $A$ approaches zero.

For small values of $A$ and $\lambda=1$ we can do the power-series expansion of Eq. (57) and we obtain

$$
\begin{aligned}
B_{2}(A)=- & \frac{359}{3990}+\frac{1851181}{67925760} A^{2}+\ldots \\
= & -0.0899749+0.027253 A^{2}+\ldots
\end{aligned}
$$

and

$$
\lim _{A \rightarrow 0} B_{2}(A)=-\frac{359}{3990}=-0.0899749
$$

which practically coincides with the value obtained from the exact solution of the cubic equation (Eq. (50)).

In Table 6 we present the relative error of the approximate frequency $\omega_{2}(A)$ when the exact expression of $B_{2}$ (solution of the cubic equation, Eq. (25)) and the approximate expression of $B_{2}$ given in Eq. (57) are used. The values of $B_{2}$ have been also included. We have only consider $\lambda=1$ because the relative errors are the highest for this value of $\lambda$ (see Table 5). As we can see both relative errors are more similar and this implies that the approximate (and simpler) expression for $B_{2}$ given in Eq. (57) is adequate for approximately solve this nonlinear oscillator by using the rational harmonic balance method. 
Table 6 Relative errors of the approximate frequencies $\omega_{2}(A)$ if we consider the exact (solution of the cubic equation, Eq. (25)) and the approximate expressions for $B_{2}$ (given in Eq. (57)) are used. The values of $B_{2}$ have been also included $(\lambda=1)$.

\begin{tabular}{llrlrrl}
\hline \multicolumn{1}{c}{$A$} & $\boldsymbol{B}_{2}$ (Eq. (25)) & $\omega_{2}(A)$ & $\begin{array}{l}\text { Relative } \\
\text { error (\%) }\end{array}$ & $B_{2}$ (Eq. (57)) & $\omega_{2}(A)$ & $\begin{array}{l}\text { Relative } \\
\text { error }(\%)\end{array}$ \\
\hline 0.1 & -0.0897435 & 0.05973115 & 0.0012 & -0.0897064 & 0.05973167 & 0.0012 \\
0.4 & -0.0859630 & 0.22907153 & 0.040 & -0.0859325 & 0.22907312 & 0.040 \\
0.7 & -0.0791806 & 0.37048459 & 0.088 & -0.0791595 & 0.37048625 & 0.088 \\
1 & -0.0713778 & 0.48029191 & 0.12 & -0.0713647 & 0.48029313 & 0.12 \\
4 & -0.0280031 & 0.83710095 & 0.038 & -0.0280029 & 0.83710096 & 0.038 \\
7 & -0.0162932 & 0.90667190 & 0.013 & -0.0162931 & 0.90667190 & 0.013 \\
10 & -0.0113491 & 0.93489862 & 0.0063 & -0.0113491 & 0.93489862 & 0.0063 \\
\hline
\end{tabular}

Figure 6 shows the relative error of the second-order approximate frequency given in Eq. (59) as a function of $A$ for $\lambda=1$. As we can see the discrepancy of the approximate frequency with the exact one never exceeds $0.12 \%$ (for $\omega_{1}(A)$ this discrepancy is as high as $2.2 \%[21])$

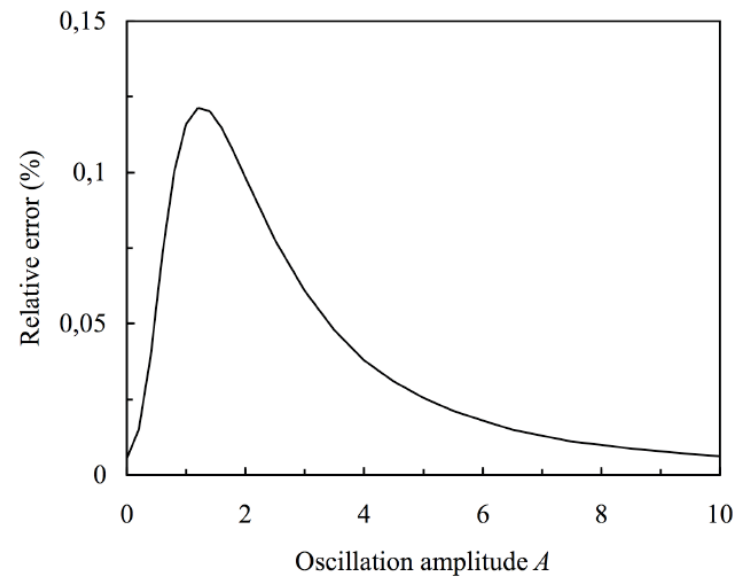

Fig. 6 Relative error of the second-order approximate frequency given in Eq. (59) as a function of $A$ for $\lambda=1$.

\section{Conclusions}

In summary, a modified rational harmonic balance method has been presented for analytically solving the oscillator typified as a mass attached to a stretched elastic wire. The major conclusion is that this scheme provides excellent approximations to the solution of this nonlinear system with high accuracy. A new procedure for solving the cubic equation for $B_{2}$ is presented and discussed. The analytical representations obtained using this technique give excellent approximations to the exact solutions for the whole range of values of oscillation amplitude $A$ and parameter $\lambda$, including the limiting cases of oscillation amplitude approaching zero and infinity. The discrepancy between the approximate frequency and the exact one never exceed $0.12 \%$. The results presented in this paper are the best reported for this type of nonlinear oscillator at the present time and they conform the future application of this modified rational harmonic balance method for other conservative nonlinear oscillators.

\section{References}

[1] R. E. Mickens, Oscillations in Planar Dynamics Systems (World Scientific, Singapore 1996).

[2] J. H. He, Some asymptotic methods for strongly nonlinear equations, Int. J. Mod. Phys. B, 20(2006), 1141-1199.

[3] A. H. Nayfeh, Problems in Perturbations (Wiley, New York 1985).

[4] P. Amore. A. Raya and F. M. Fernández, Comparison of alternative improved perturbative methods for nonlinear oscillations, Phys. Lett. A, 340(2005), 201-208.

[5] J. H. He, Modified Lindstedt-Poincare methods for some non-linear oscillations. Part I: expansion of a constant, Int. J. Nonlin. Mech., 37(2002), 309-314.

[6] J. H. He, Variational approach for nonlinear oscillators, Chaos Soliton. Fract., 34 (2007), 1430-1439. 
[7] Mo. Miansari, D. D. Ganji and Me. Miansari, Application of He's variational iteration method to nonlinear heat transfer equations, Phys. Lett. A, 372(2008), 779-785.

[8] J. H. He, X. H. Wu, Construction of solitary solution and compact on-like solution by variational iteration method, Chaos Soliton. Fract., 29(2006), 108-113.

[9] M. Dehghan and M. Tatari, The use of He's variational iteration method for solving multipoint boundary value problems, Phys. Scripta,. 74 (2007), 672-676.

[10] M. Tatari, M. Dehghan, On the convergence of He's variational iteration method, $J$. Comput. Appl. Math., 207(2007), 121-128.

[11] J. H. He, Homotopy perturbation method for bifurcation on nonlinear problems, Int. J. Nonlin. Sci. Num., 6(2005), 207-208.

[12] A. Beléndez, A. Hernández, T. Beléndez, E. Fernández, M. L. Álvarez and C. Neipp, Application of He's homotopy perturbation method to the Duffing-harmonic oscillator, Int. J. Nonlin. Sci. Num., 8(2007), 79-88.

[13] M. S. H. Chowdhury and I. Hashim, Application of homotopy-perturbation method to nonlinear population dynamics models, Phys. Lett. A, 368(2007), 251-258.

[14] A. Beléndez, A.Hernández, T.Beléndez, et al., Application of the homotopy perturbation method to the nonlinear pendulum, Eur.J.Phys.,28(2007), 93-104.

[15] D. D. Ganji and A. Sadighi, Application of He's homotopy-perturbation method to nonlinear coupled systems of reaction-diffusion equations, Int. J. Nonlin. Sci. Num., 7(2006), 411-418.

[16] T. Özis and A. Yildirim, A comparative study of He's homotopy perturbation method for determining frequency-amplitude relation of a nonlinear oscillator with discontinuities, Int. J. Nonlin. Sci. Num., 8(2007), 243-248.

[17] J. H. He, An elementary introduction to recently developed asymptotic methods and nanomechanics in textile engineering, Int. $J$. Mod. Phys. B, 22 (2008), 3487-3578.

[18] A. Beléndez and C. Pascual, Harmonic balance approach to the periodic solutions of the (an)harmonic relativistic oscillator, Phys. Lett. A, 371(2007), 291-299.

[19] H. Hu, J. H. Tang, Solution of a Duffing-harmonic oscillator by the method of harmonic balance, J. Sound Vib., 294(2006), 637-639.
[20] C. W. Lim and B. S. Wu, A new analytical approach to the Duffing-harmonic oscillator, Phys. Lett. A, 311(2003), 365-373.

[21] A. Beléndez, A. Hernández, T. Beléndez, M. L. Álvarez, S. Gallego, M. Ortuño and C. Neipp, Application of the harmonic balance method to a nonlinear oscillator typified by a mass attached to a stretched wire, J. Sound Vib., 302(2007), 1018-1029.

[22] A. Beléndez, A. Hernández, A. Márquez, T. Beléndez and C. Neipp, Analytical approximations for the period of a simple pendulum, Eur. J. Phys. 27(2006), 539-551.

[23] C. W. Lim, B. S. Wu and W. P. Sun, Higher accuracy analytical approximations to the Duffing-harmonic oscillator, J. Sound Vib. 296(2006), 1039-1045.

[24] B. S. Wu, W. P. Sun and C. W. Lim, An analytical approximate technique for a class of strongly non-linear oscillators, Int. $J$. Non-Linear Mech. 41(2006), 766-774.

[25] A. Beléndez, T. Beléndez, C. Neipp, et al., Approximate solutions of a nonlinear oscillator typified as a mass attached to a stretched elastic wire by homotopy perturbation method, Chaos Soliton. Fract., 37(2008), 770-780.

[26] W. P. Sun, B. S. Wu,C. W. Lim, Approximate analytical solutions for oscillation of a mass attached to a stretched elastic wire, J. Sound Vib., 300(2007), 1042-1047.

[27] L. Xu, Application of He's parameterexpansion method to an oscillation of a mass attached to a stretched elastic wire, Phys. Lett. A, 368(2007), 259-262.

[28] D. H. Shou, Variational approach to the nonlinear oscillator of a mass attached to a stretched wire, Phys. Scripta.77(2008), art. 045006.

[29] R. E. Mickens and D. Semwogerere, Fourier analysis of a rational harmonic balance approximation for periodic solutions, J. Sound Vib., 195 (1996), 528-530.

[30] A. Beléndez, E. Gimeno, E. Fernández, D. I. Méndez and M. L. Álvarez, Accurate approximate solution to nonlinear oscillators in which the restoring force is inversely proportional to the dependent variable, Phys. Scripta. 77(2008), art. 065004. 